\title{
HAUSDORFF CONTENT AND RATIONAL APPROXIMATION IN FRACTIONAL LIPSCHITZ NORMS
}

BY

\author{
ANTHONY G. O'FARRELL( $\left.{ }^{l}\right)$
}

\begin{abstract}
For $0<\alpha<1$, we characterise those compact sets $X$ in the plane with the property that each function in the class $\operatorname{lip}(\alpha, X)$ that is analytic at all interior points of $X$ is the $\operatorname{limit}$ in $\operatorname{Lip}(\alpha, X)$ norm of a sequence of rational functions. The characterisation is in terms of Hausdorff content.
\end{abstract}

1. If $E$ is a closed subset of the complex plane $\mathbf{C}$, and $f$ is a bounded complex-valued function on $E$ we define the modulus of continuity $\omega_{f}$ by setting

$$
\omega_{f}(r)=\sup \{|f(x)-f(y)|: x, y \in E,|x-y| \leqslant r\}
$$

whenever $r \geqslant 0$. Thus $\omega_{f}$ is a nondecreasing function, $\omega(0)=0$, and $f$ is uniformly continuous on $E$ if and only if $\omega_{f}$ is continuous at zero. For $0<\alpha<1$ we define

$$
\begin{aligned}
\|f\|_{\alpha, E} & =\sup \left\{r^{-\alpha} \omega_{f}(r): r>0\right\}, \\
\operatorname{Lip}(\alpha, E) & =\left\{f:\|f\|_{\alpha, E}<\infty\right\}, \\
\operatorname{lip}(\alpha, E) & =\left\{f \in \operatorname{Lip}(\alpha, E): r^{-\alpha} \omega_{f}(r) \rightarrow 0 \text { as } r \downarrow 0\right\} .
\end{aligned}
$$

When given the norm

$$
\|f\|_{\alpha, E}^{\prime}=\|f\|_{\alpha, E}+\|f\|_{u, E}
$$

(where $\|f\|_{u, E}$ is the sup norm), $\operatorname{Lip}(\alpha, E)$ becomes a Banach algebra, and $\operatorname{lip}(\alpha, E)$ is a closed point-separating subalgebra [9]. This paper concerns the question of approximation in $\operatorname{Lip}(\alpha, X)$, for compact sets $X$, by rational functions with poles off $X$.

Before stating the main result, we must define the Hausdorff contents $M^{\beta}$ and $M_{*}^{\beta}$. A measure function is a nonnegative increasing function defined on

Received by the editors May 5, 1974 and, in revised form, April 14, 1975.

AMS (MOS) subject classifications (1970). Primary 30A82, 46J99.

Key words and phrases. Capacity, complex plane.

(') This work was supported by NSF GP 33693X. 
$\mathbf{R}^{+}=\{t \in \mathbf{R}: t \geqslant 0\}$. If $h$ is a measure function and $F \subset \mathbf{C}$, then the Hausdorff content $M_{h}(F)$ is the infimum of all sums

$$
\sum_{S \in \mathcal{S}} h(\operatorname{diam} S)
$$

where $\mathcal{S}$ runs over all countable coverings of $F$ by closed (or open) balls. In case $h(r)=r^{\beta}$ for some $\beta>0$, we write $M_{h}=M^{\beta}$. The set function $M_{*}^{\beta}$ is defined by setting

$$
\begin{aligned}
& M_{*}^{\beta}(F)=\sup \left\{M_{h}(F): h\right. \text { is a measure function, } \\
& \left.\qquad h(r) \leqslant r^{\beta}, r^{-\beta} h(r) \rightarrow 0 \text { as } r \downarrow 0\right\} .
\end{aligned}
$$

Theorem. Let $X$ be a compact subset of $\mathbf{C}$, and let $0<\alpha<1$. In order that every function in $\operatorname{lip}(\alpha, X)$ which is analytic on the interior of $X$ be the limit in $\operatorname{Lip}(\alpha, X)$ norm of a sequence of rational functions, it is necessary and sufficient that there exist a constant $\mu>0$ such that

$$
M^{1+\alpha}(D \backslash X) \geqslant \mu M_{*}^{1+\alpha}(D \backslash \text { int } X)
$$

whenever $D$ is an open disc.

It is worth noting that the condition for approximation is purely metric, in contrast to the conditions which have been obtained for uniform approximation [12].

The necessity of the condition is proved in $\S \S 2-8$. We introduce capacities in $\$ 2$ and show that if two spaces have the same closure then the corresponding capacities coincide. In \$\$3-7 we apply a generalisation of Melnikov's Theorem [10] in order to relate the capacities corresponding to rational functions and lip $\alpha$ analytic functions to the contents $M^{1+\alpha}$ and $M_{*}^{1+\alpha}$. The proof of sufficiency in $\$ \S 10-15$ is modelled on the Vitushkin approximation scheme [12], [6], [8] as modified by Davie [3]. We make heavy use of the metric character of the capacities. We give some applications in \$§16-23.

Throughout the paper, $\alpha$ is fixed, $0<\alpha<1$; $\mathbf{Z}$ denotes the set of integers, and $\mathbf{Z}^{+}=\mathbf{Z} \cap \mathbf{R}^{+} ; \Sigma$ is the Riemann sphere; $\mathscr{D}$ is the space of complex-valued $C^{\infty}$ functions with compact support. If $f$ is continuous on $\mathbf{C}$ and $\varphi \in \mathscr{D}$ we define

$$
T_{\varphi} f(z)=\frac{1}{\pi} \int \frac{f(z)-f(\zeta)}{z-\zeta} \frac{\partial \varphi}{\partial \bar{\zeta}} d m(\zeta),
$$

where $m$ denotes Lebesgue measure on the plane. For an exposition of the properties of this " $T_{\varphi}$-operator", see [6]. A set $B$ of continuous functions on $\mathbf{C}$ is said to be $T$-invariant if $T_{\varphi} f \in B$ whenever $f \in B$ and $\varphi \in \mathscr{D}$. The operator $T_{\varphi}$ is bounded with respect to the $\operatorname{Lip}(\alpha, \mathbf{C})$ norm, for each $\varphi \in \mathscr{D}$. 
In fact

$$
\left\|T_{\varphi} f\right\|_{\alpha, \mathbf{C}} \leqslant K \eta_{f}(d)\left\{\|\varphi\|_{u}+d\|\nabla \varphi\|_{u}\right\}
$$

where $K$ is a constant depending only on $\alpha$,

$$
d=\operatorname{diam} \operatorname{spt} \varphi, \quad \eta_{f}(d)=\sup \left\{s^{-\alpha} \omega_{f}(s): 0<s \leqslant d\right\} .
$$

The symbol $X$ always stands for a compact subset of $\mathbf{C}, \mathcal{R}(X)$ is the subspace of $\operatorname{Lip}(\alpha, C)$ consisting of those functions which agree on some neighbourhood of $X$ with a rational function, and $\tilde{\Re}(X)$ is the space of functions in $\operatorname{Lip}(\alpha, C)$ which are analytic on a neighbourhood of $X$. If $B$ is any subspace of $\operatorname{Lip}(\alpha, X)$, then the closure of $B$ with respect to the norm $\|\cdot\|_{\alpha, X}^{\prime}$ is denoted $[B]_{\alpha, X}$, or just $[B]_{\alpha}$. If $B$ contains the constants, then this coincides with the closure with respect to the norm $\|\cdot\|_{\alpha, X}$. For any $X$,

$$
[\Re(X)]_{\alpha, X}=[\tilde{R}(X)]_{\alpha, X} \text {. }
$$

This assertion is the $\alpha$ version of Runge's Theorem, and the classical proof of Runge's Theorem is easily modified to prove it.

As a technical convenience, we assume that the diameter of $X$ does not exceed $\frac{1}{4}$.

I am grateful to T. Gamelin and J. Garnett for valuable conversations. The Decay Lemma of $\S 12$ is the work of Garnett. I am grateful to the referee for suggesting the argument of $\$ 4$.

2. We follow established custom in denoting the algebra of all continuous complex-valued functions on $X$ by $C(X)$ and denoting the subalgebra of functions analytic on $\operatorname{int}(X)$ by $A(X)$. We further define

$$
A^{\alpha}(X)=\operatorname{Lip}(\alpha, X) \cap A(X), \quad A_{\alpha}(X)=\operatorname{lip}(\alpha, X) \cap A(X),
$$

so that $A^{\alpha}$ and $A_{\alpha}$ are closed subalgebras of Lip $\alpha$. In view of the extension theorem [11, Chapter VI], a subspace $V \subset A^{\alpha}(X)$ may be regarded as a subspace of $\operatorname{Lip}(\alpha, C)$ (we may identify $V$ with the set of functions in $\operatorname{Lip}(\alpha, \mathbf{C})$ whose restrictions to $X$ lie in $V$ ), so $T$-invariance makes sense for such subspaces. To each $T$-invariant subspace $V$ of $A^{\alpha}(X)$ we associate a capacity $\gamma(V, \circ)$, a nonnegative increasing function defined on the family $\{D\}$ of open discs: we say a function $f \in V$ is $D$-admissible if $f$ is analytic off a compact subset of $D, f(\infty)=0$, and $\|f\|_{\alpha, C} \leqslant 1$; we set

$$
\gamma(V, D)=\sup \left\{\left|f^{\prime}(\infty)\right|: f \in V, f \text { is } D \text {-admissible }\right\}
$$

Lemma. Let $V$ and $W$ be $T$-invariant subspaces of $A^{\alpha}(X)$. Suppose $V$ and $W$ have the same closure in $\operatorname{Lip}(\alpha, X)$ norm. Then $\gamma(V, D)=\gamma(W, D)$ for every open disc $D$.

Proof. It suffices to show that 


$$
\gamma(V, D)=\gamma\left([V]_{\alpha}, D\right)
$$

It is clear that

$$
\gamma(V, D) \leqslant \gamma\left([V]_{\alpha}, D\right)
$$

To prove the opposite inequality, let $D$ be a fixed open disc and let $\varepsilon>0$ be given. Choose $f \in[V]_{\alpha}$ such that $f$ is $D$-admissible and

$$
\left|f^{\prime}(\infty)\right|>\gamma\left([V]_{\alpha}, D\right)-\varepsilon .
$$

Choose a sequence $\left\{f_{n}\right\}_{1}^{\infty}$ of elements of $V$ such that $\left\|f_{n}-f\right\|_{\alpha, X} \rightarrow 0$. For each $n$ the extension theorem ensures the existence of a function

$$
g_{n} \in \operatorname{Lip}(\alpha, \mathbf{C})
$$

such that $g_{n}=f_{n}-f$ on $X$ and $\left\|g_{n}\right\|_{\alpha, \mathrm{C}} \leqslant 4\left\|f_{n}-f\right\|_{\alpha, X}$. Let $h_{n}=f+g_{n}$. Then $h_{n} \in V$ and $\left\|h_{n}-f\right\|_{\alpha, \mathrm{C}} \rightarrow 0$ as $n \rightarrow+\ldots$ Choose $\varphi \in \mathcal{D}$ such that spt $\varphi \subset D$ and $\varphi \equiv 1$ on a neighbourhood of the set of singularities of $f$. Then $T_{\varphi} f=f, T_{\varphi} h_{n} \in V$, and

$$
\begin{aligned}
\left\|T_{\varphi} h_{n}-f\right\|_{\alpha, \mathrm{C}} & =\left\|T_{\varphi}\left(h_{n}-f\right)\right\|_{\alpha, \mathrm{C}} \\
& <K\left\|h_{n}-f\right\|_{\alpha, D}\left\{\|\varphi\|_{u}+\operatorname{diam} D\|\nabla \varphi\|_{u}\right\},
\end{aligned}
$$

by $\S 1$. Thus $\left\|T_{\varphi} h_{n}-f\right\|_{\alpha, \mathrm{C}} \rightarrow 0$, and hence $\left(T_{\varphi} h_{n}\right)^{\prime}(\infty) \rightarrow f^{\prime}(\infty)$, so that

$$
\gamma(V, D) \geqslant \gamma\left([V]_{\alpha}, D\right)-\varepsilon \text {. }
$$

Since this holds for each $\varepsilon>0$, we conclude that (*) holds.

We do not know whether or not the converse to this lemma is true in general.

3. In order to apply Lemma 2 to rational approximation we have to describe the capacities $\gamma(V, \cdot)$ in the cases $V=\Re(X)$ and $V=A_{\alpha}(X)$. Melnikov's Theorem provides the key. It relates certain capacities to the Hausdorff contents $M_{h}$. Before stating it we define a special class of "modulus of continuity functions".

Consider a concave increasing function $\omega(r)$, defined for $r \geqslant 0$ and constant for $r \geqslant 1$, with $\omega(0)=0$, and such that

(1) $\omega^{\prime}(r)$ exists for $r>0$;

(2) there exists a constant $L_{1}>0$ such that $\omega(r) \leqslant L_{1} r \omega^{\prime}(r)$ for $0<r<\frac{1}{2}$;

(3) there exists a constant $L_{2}>1$ such that $r \omega^{\prime}(r) \leqslant\left(L_{2}-1\right) \omega(r) / L_{2}$ for $0<r<\frac{1}{2}$.

Such a $\omega$ we call a modulated function. To each modulated function is associated a measure function $h$, defined by $h(r)=r \omega(r)$, and a capacity $\tau(\omega, \cdot)$ defined on arbitrary bounded sets $E \subset \mathbf{C}$ by

$\tau(\omega, E)=\sup \left\{\left|f^{\prime}(\infty)\right|: f\right.$ is analytic on a neighbourhood of

$$
\left.\Sigma \backslash E, f(\infty)=0, \omega_{f} \leqslant \omega\right\}
$$


Here $\omega_{f}$ refers to the modulus of continuity of $f$ as a function on $\mathbf{C}$.

Melnikov's TheOREM. Let $\omega$ be a modulated function. Then there is a constant $K(\omega)$ such that

$$
K^{-1} M_{h}(E) \leqslant \tau(\omega, E) \leqslant K M_{h}(E)
$$

whenever $E$ is compact or $E$ is open and bounded. $K(\omega)$ may be taken to be $K_{0}\left(L_{1}+L_{2}\right)$, where $K_{0}$ is a certain universal constant.

Actually, this is a slight extension of Melnikov's result. He proved it in case $\omega(r)=r^{\beta}$ for some $\beta, 0<\beta<1$, and in that case $K(\omega)$ may be taken to be $K_{0} \beta^{-1}(1-\beta)^{-1}$. His proof [10] carries over with trivial changes. We omit the details.

An example of a modulated function other than the various $r^{\beta}, 0<\beta<1$, is obtained by fixing $0<\delta<1$ and setting

$$
\omega(r)= \begin{cases}r^{\delta}\left\{\delta^{-1}-\log 2 r\right\}, & 0<r<\frac{1}{2}, \\ \delta^{-1} 2^{-\delta}, & \frac{1}{2} \leqslant r<\infty .\end{cases}
$$

4. LEMMA. Let $\omega(r)$ be a nonnegative function such that $\omega(r) \leqslant r^{\alpha}$ and $r^{-\alpha} \omega(r) \rightarrow 0$. Let $\varepsilon>0$ and $\beta>\alpha$ be given. Then there exists a modulated function $\omega_{1}(r)$ with the following properties:

(1) $(1-\varepsilon) \omega(r) \leqslant \omega_{1}(r) \leqslant r^{\alpha}$ for $0 \leqslant r \leqslant \frac{1}{2}$,

(2) $\alpha \omega_{1}(r) \leqslant r \omega_{1}^{\prime}(r) \leqslant \beta \omega_{1}(r)$ for $0 \leqslant r \leqslant \frac{1}{2}$,

(3) $r^{-\alpha} \omega_{1}(r) \rightarrow 0$ as $r \downarrow 0$.

Proof. In proving this, we may suppose that $\beta<\alpha(1-\varepsilon)^{-1}$. Choose a monotonically-decreasing sequence of piecewise smooth functions $\psi_{j}$ such that

(4) $\beta(1-\varepsilon) \omega(r) / \alpha \leqslant \psi_{j}(r) \leqslant r^{\alpha}$,

(5) $\alpha \psi_{j}(r) \leqslant r \psi_{j}^{\prime}(r) \leqslant \beta \psi_{j}(r)$,

(6) $\psi_{j}(r)<r^{\alpha} / j$ in a neighbourhood of the origin.

Such $\psi_{j}$ 's may be constructed as follows: Choose $\delta_{j}>\alpha$, put

$$
\begin{aligned}
& \varphi_{j}(r)=\max \left\{r^{\alpha} / j, r^{\delta_{j}}\right\}, \quad \text { and } \\
& \psi_{j}(r)=\min \left\{\alpha \int_{0}^{r} \frac{\varphi_{j}(s)}{s} d s, \psi_{j-1}(r)\right\} .
\end{aligned}
$$

If $\delta_{j}$ is sufficiently close to $\alpha$, properties (4), (5) and (6) are satisfied, as is seen by a routine calculation.

Set $\varphi(r)=\lim \psi_{j}(r)$. It follows easily that

$$
\omega_{1}(r)=\alpha \int_{0}^{r} \frac{\varphi(s)}{s} d s
$$


satisfies properties (1), (2), and (3). Verification is again routine. This completes the proof.

Fix $\beta=(1+\alpha) / 2$. For each $f \in \operatorname{lip}(\alpha, \mathbf{C})$ with $\|f\|_{\alpha} \leqslant 1$, and each $\varepsilon>0$, choose a modulated function $\omega_{1}(r)$ such that

$$
\begin{aligned}
& (1-\varepsilon) \omega_{f}(r) \leqslant \omega_{1}(r) \leqslant r^{\alpha}, \\
& \alpha \omega_{1}(r) \leqslant r \omega_{1}^{\prime}(r) \leqslant \beta \omega_{1}(r), \\
& r^{-\alpha} \omega_{1}(r) \rightarrow 0 \text { as } r \downarrow 0 .
\end{aligned}
$$

Let $\mathscr{F}_{\alpha}$ denote the family of all functions $\omega_{1}$ obtained in this way. Clearly, we may apply Melnikov's Theorem to all $\omega_{1} \in \mathscr{F}_{\alpha}$ at once, using the same constant $K$.

5. Corollary. Let $X \subset \mathrm{C}$ be compact, $V=\tilde{\Re}(X)$. Then for all open discs D

$$
K^{-1} \gamma(V, D) \leqslant M^{1+\alpha}(D \backslash X) \leqslant K \gamma(V, D)
$$

where $K$ depends only on $\alpha$.

Proof. Choose a sequence of open sets $\left\{U_{n}\right\} \downarrow X$ such that each set bdy $\left(U_{n}\right)$ is a finite union of smooth curves. Then

$$
M^{1+\alpha}(D \backslash X)=\lim _{n \uparrow \infty} M^{1+\alpha}\left(D \backslash U_{n}\right) .
$$

Next, for $n=1,2,3, \ldots$, we have

$$
A^{\alpha}\left(X_{n}\right) \subset V \subset \bigcup_{m=1}^{\infty} A^{\alpha}\left(X_{m}\right),
$$

where $X_{n}=\operatorname{clos}\left(U_{n}\right)$. Hence for each open $\operatorname{disc} D$,

$$
\gamma\left(A^{\alpha}\left(X_{n}\right), D\right) \leqslant \gamma(V, D) \leqslant \lim _{m \uparrow \infty} \gamma\left(A^{\alpha}\left(X_{m}\right), D\right) .
$$

Applying Melnikov's Theorem with $\omega(r)=r^{\alpha}$ and $E=D \backslash X_{n}$ (so that $\left.\tau(\omega, E)=\gamma\left(A^{\alpha}\left(X_{n}\right), D\right)\right)$, we obtain

$$
K^{-1} \gamma\left(A^{\alpha}\left(X_{n}\right), D\right) \leqslant M^{1+\alpha}\left(D \backslash X_{n}\right) \leqslant K \gamma\left(A^{\alpha}\left(X_{n}\right), D\right),
$$

for $n=1,2,3, \ldots$, where $K$ depends only on $\alpha$. Taking limits we get the desired result.

6. In the definition of $M_{*}^{1+\alpha}$ it suffices to consider those $h$ of the form $r \omega(r)$ for $\omega \in \mathscr{F}_{\alpha}$.

7. Corollary. Let $W=A_{\alpha}(X)$. Then for all open discs $D$,

$$
K^{-1} \gamma(W, D) \leqslant M_{*}^{1+\alpha}(D \backslash \text { int } X) \leqslant K \gamma(W, D),
$$

where $K$ depends only on $\alpha$.

Proof. Let $f \in W$ be $D$-admissible, and let $\varepsilon>0$ be given. Then there exists $\omega \in \mathcal{F}_{\alpha}$ such that $(1-\varepsilon) \omega_{f} \leqslant \omega$. Thus 


$$
(1-\varepsilon)\left|f^{\prime}(\infty)\right| \leqslant \tau(w, D \backslash \text { int } X) .
$$

If $h(r)=r \omega(r)$, then Melnikov's Theorem yields

$$
\tau(\omega, D \backslash \text { int } X) \leqslant K(\omega) M_{h}(D \backslash \text { int } X) .
$$

Thus

$$
(1-\varepsilon) \gamma(W, D) \leqslant K M_{*}^{1+\alpha}(D \backslash \text { int } X),
$$

where $K=\sup \left\{K_{0}\left(L_{1}+L_{2}\right): \omega \in \mathscr{F}_{\alpha}\right\}$ depends only on $\alpha$. This proves the first inequality.

For the second, fix $\omega \in \mathscr{F}_{\alpha}$, and let $h(r)=r \omega(r)$. Let $f \in C(\Sigma)$ be analytic off $(D \backslash$ int $X)$, with $\omega_{f} \leqslant \omega, f(\infty)=0$. Then $f \in W$ and $f$ is $D$-admissible. Hence $\left|f^{\prime}(\infty)\right| \leqslant \gamma(W, D)$. Thus $\tau(\omega, D \backslash$ int $X) \leqslant \gamma(W, D)$. By Melnikov's Theorem

$$
K(\omega)^{-1} M_{h}(D \backslash \text { int } X) \leqslant \gamma(W, D) .
$$

Since this holds for every $\omega \in \mathscr{F}_{\alpha}$, we conclude that

$$
K^{-1} M_{*}^{1+\alpha}(D \backslash \operatorname{int} X) \leqslant \gamma(W, D),
$$

with $K$ as above.

8. Combining the results of $\S \S 1,2,5$, and 7 , we deduce the necessity of the condition of the theorem. In fact, if $[\Re]_{\alpha}=A_{\alpha}(X)$, then

$$
M^{1+\alpha}(D \backslash X) \geqslant K M_{*}^{1+\alpha}(D \backslash \text { int } X),
$$

for every open disc $D$, where $K>0$ is a constant which depends only on $\alpha$.

9. RemarK. One might wonder whether it is always possible, given a modulated function $\omega$, to find functions $f \in A(X)$ such that $\omega_{f} \leqslant \omega$ but $\omega(r)^{-1} \omega_{f}(r) \nrightarrow 0$ as $r \rightarrow 0$. Putting it another way, if $\omega_{1}(r) \omega_{2}(r)^{-1} \rightarrow 0$ as $r \rightarrow 0$, are there any functions $f$ in $A(X)$ such that $\omega_{f} \leqslant \omega_{2}$ but $\omega_{f} \neq o\left(\omega_{1}\right)$ ? The answer is yes. This follows from some results of Dolženko [4].

10. the first step towards proving the sufficiency of the approximation condition is a lemma which gives an estimate for the uniform norm in terms of the Lip $\alpha$ norm.

Lemma. Suppose $E \subset \mathrm{C}$ is bounded, $f$ is analytic on, $\Sigma \backslash E, f(\infty)=0$, and $f \in \operatorname{Lip}(\alpha, \mathbf{C})$. Then

$$
\|f\|_{u, \mathbf{C}} \leqslant 2^{1+\alpha}(\operatorname{diam} E)^{\alpha}\|f\|_{\alpha, \mathbf{C}}
$$

Proof. There is a circle $C$ of radius diam $E$ which encloses $E$. Since $f(\infty)=0$, then $\int_{C} f d v=0$. Hence, if $f=u+i v$, then $\int_{C} u d v=\int_{C} v d \vartheta=0$. Thus $u$ and $v$ each have a zero on $C$. Thus for $x$ inside $S$,

$$
|u(x)| \leqslant(2 \operatorname{diam} E)^{\alpha}\|f\|_{\alpha}, \quad|v(x)| \leqslant(2 \operatorname{diam} E)^{\alpha}\|f\|_{\alpha},
$$

hence 


$$
|f(x)| \leqslant 2^{1+\alpha}(\operatorname{diam} E)^{\alpha}\|f\|_{\alpha^{\prime}}
$$

and the result follows by the maximum principle.

The above estimate is somewhat crude, in that it depends only on the diameter of $E$. A more refined version is obtain in $\$ 14$.

11. Now fix $X$ compact in $\mathbf{C}$ and abbreviate $\Re=\Re(X), A=A_{\alpha}(X)$, $\gamma(D)=\gamma(\Re, D), \gamma_{A}(D)=\gamma(A, D)$. Let $c(D)$ denote the centre of the disc $D$, and let $\tau D$ denote the disc with centre $c(D)$ and radius equal to $\tau$ times the radius of $D$. For any function $f$ which is analytic on a neighbourhood of $\infty$ we may write

$$
f(z)=a_{0}+\frac{a_{1}}{z-c(D)}+\frac{a_{2}}{(z-c(D))^{2}}+\ldots
$$

for large $z$. Here $a_{0}=f(\infty), a_{1}=f^{\prime}(\infty)$, and we define $\beta(f, D)=a_{2}$. If $a_{0}=a_{1}=0$, then $\beta(f, D)$ does not depend on $D$.

LEMMA. Let $D$ be an open disc of radius $r$, and let $f \in \Re$ be $D$-admissible. Then

$$
|\beta(f, D)| \leqslant \operatorname{Kr} \gamma(D)
$$

where $K$ is a constant depending only on $\alpha$. For $f \in A$ the same inequality holds, but with $\gamma$ replaced by $\gamma_{A}$.

Proof. Let $f \in \Re$ be $D$-admissible. Then $f$ is analytic off $D, f(\infty)=0$, and $\|f\|_{\infty} \leqslant 1$. We define the function $g \in \Re$ by setting

$$
g(z)=(z-c(D)) f(z)-f^{\prime}(\infty) .
$$

Then $g(\infty)=0, g^{\prime}(\infty)=\beta(f, D)$, and we claim that $\|g\|_{\alpha} \leqslant K_{8} r$, where $K_{8}$ depends only on $\alpha$.

In proving this claim we may assume $c(D)=0$. Let $z, w \in \mathbf{C}, z \neq w$. We consider four cases, which together cover all the possiblilites.

Case $1 . z, w \in 3 D$. Then

$$
\begin{aligned}
\frac{|z f(a)-w f(w)|}{|z-w|^{\alpha}} & \leqslant \frac{|z||f(z)-f(w)|+|z-w||f(w)|}{|z-w|^{\alpha}} \\
& \leqslant 3 r\|f\|_{\alpha}+(6 r)^{1-\alpha}\|f\|_{u} \\
& <K_{1} r\|f\|_{\alpha} \text { by } \S 10 \\
& \leqslant K_{1} r .
\end{aligned}
$$

Case 2. $z, w \in \mathbf{C} \backslash 2 D,|z-w| \geqslant r$. Then 


$$
\begin{aligned}
& \frac{|z f(z)-w f(w)|}{|z-w|^{\alpha}} \leqslant \frac{|z f(z)|}{r^{\alpha}}+\frac{|w f(w)|}{r^{\alpha}} \\
& \quad<2 r^{1-\alpha}(|f(z)|+|f(w)|) \leqslant K_{1} r^{1-\alpha}\left\{\frac{r\|f\|_{u}}{|z|}+\frac{r\|f\|_{u}}{|w|}\right\} \\
& \quad<K_{2} r^{1-\alpha}\|f\|_{u} \leqslant K_{3} r\|f\|_{\alpha} \leqslant K_{3} r .
\end{aligned}
$$

In the third inequality we used the uniform norm decay estimate [6, p. 201], and in the fifth we again applied $\$ 10$.

Case 3. $z, w \in \mathbf{C} \backslash 2 D,|z-w|<r$. Then

$$
\begin{aligned}
& \frac{|z f(z)-w f(w)|}{|z-w|^{\alpha}} \\
& \quad=\frac{1}{|z-w|^{\alpha}}\left|\frac{1}{2 \pi i} \int_{|\zeta|=r} \zeta f(\zeta)\left\{\frac{1}{\zeta-z}-\frac{1}{\zeta-w}\right\} d \zeta\right| \\
& \quad \leqslant \frac{K_{4} r|| f \|_{u}}{|z-w|^{\alpha}} \int_{|\zeta|=r} \frac{|z-w|}{|\zeta-z||\zeta-w|}|d \zeta| \\
& \quad \leqslant K_{5} r^{1+\alpha}\|f\|_{\alpha}|z-w|^{1-\alpha} r^{-1}<K_{5} r .
\end{aligned}
$$

Case 4. $z \in 2 D, w \notin 3 D$. Then

$$
\begin{aligned}
\frac{|z f(z)-w f(w)|}{|z-w|^{\alpha}} & \leqslant \frac{|z f(z)|}{r^{\alpha}}+\frac{|w f(w)|}{r^{\alpha}} \\
& \leqslant 2 r^{1-\alpha}\|f\|_{u}+\frac{|w|}{r^{\alpha}} \cdot \frac{r\|f\|_{u}}{|w|} \leqslant K_{6} r^{1-\alpha}\|f\|_{u} \leqslant K_{7} r .
\end{aligned}
$$

Hence the claim is true, so that $\left(K_{8} r\right)^{-1} g$ is $D$-admissible. Thus

$$
|\beta(f, D)|=\left|g^{\prime}(\infty)\right| \leqslant K_{8} r \gamma(D) .
$$

The assertion about $A$ is proved similarly.

12. Decay Lemma (GaRnetr). Let $D$ be a disc of radius $r$, and let $z \in \mathbf{C}$, with $d=\operatorname{dist}(z, D) \geqslant r$. Then

$$
|f(z)| \leqslant K \gamma(D)\|f\|_{\alpha} / d
$$

and

$$
\left|f^{\prime}(z)\right| \leqslant K \gamma(D)\|f\|_{\alpha} / d^{2}
$$

whenever $f \in \Re$. There is a similar estimate for $f \in A$, with $\gamma$ replaced by $\gamma_{A}$.

Proof. (1) $D \backslash X$ may be covered by a finite collection $\left\{S_{j}\right\}$ of open squares with sides parallel to the axes, such that 


$$
\sum\left(\text { side } S_{j}\right)^{1+\alpha}<4 M^{1+\alpha}(D \backslash X) / \pi,
$$

and no square is contained in the union of the rest. Arrange the squares in an order of nondecreasing side-lengths, and form $H_{1}=S_{1}, H_{2}=S_{2} \backslash S_{1}, H_{3}=$ $S_{3} \backslash S_{1} \backslash S_{2}$, and so on. For each $i$, let $\Gamma_{j}=$ bdy $H_{j}$, and choose $\zeta_{j} \in$ int $H_{1}$. Observe that the length of $\Gamma_{i}$ is at most $4\left(\right.$ side $\left.S_{i}\right)$. Then

$$
\begin{aligned}
|f(z)| & =\left|\frac{1}{2 \pi i} \sum_{j} \int_{\Gamma_{j}} \frac{f(\zeta)}{\zeta-z} d \zeta\right| \\
& \leqslant \frac{1}{2 \pi} \sum_{j}\left|\int_{\Gamma_{j}} \frac{f(\zeta)-f\left(\zeta_{j}\right)}{\zeta-z} d \zeta\right| \\
& \leqslant K_{1} \sum_{j} \frac{\left(\operatorname{side} S_{j}\right)^{1+\alpha}}{d}\|f\|_{\alpha} \leqslant \frac{K_{2} M^{1+\alpha}(D \backslash X)\|f\|_{\alpha}}{d} \\
& \leqslant \frac{K_{3} \gamma(D)\|f\|_{\alpha}}{d}, \text { by Corollary 5. }
\end{aligned}
$$

The estimate for $f^{\prime}(z)$ is obtained in a similar way.

To prove the corresponding estimate for $f \in A$, first choose a modulated function $\omega$ such that

$$
\begin{aligned}
\frac{1}{2} \omega_{f}(r) & \leqslant\|f\|_{\alpha} \omega(r), \quad 0 \leqslant r \leqslant \frac{1}{2}, \\
\omega(r) & \leqslant r^{\alpha}, \quad 0 \leqslant r \leqslant \frac{1}{2}, \\
r^{-\alpha} \omega(r) & \rightarrow 0 \quad \text { as } r \downarrow 0 .
\end{aligned}
$$

Set $h(r)=r \omega(r)$. An argument like that above shows that

$$
|f(z)|<K_{4} M_{h}(D \backslash \text { int } X)\|f\|_{\alpha} / d,
$$

and so

$$
|f(z)| \leqslant \frac{K_{4} M_{*}^{1+\alpha}(D \backslash \text { int } X)\|f\|_{\alpha}}{d} \leqslant \frac{K_{5} \gamma_{A}(D)\|f\|_{\alpha}}{d}, \text { by } \S 7 .
$$

13. LemMA. Let $D$ be an open disc, $s^{1+\alpha}=M^{1+\alpha}(D \backslash X)$, and let $\left\{B_{j}\right\}$ be a family of discs of radius $s$, each of which is contained in $D$, such that no point belongs to more than $p$ of the $B_{j}$. Then there is a constant $K$, depending only on $\alpha$, such that

$$
\sum_{j} M^{1+\alpha}\left(B_{j} \backslash X\right) \leqslant K p M^{1+\alpha}(D \backslash X)
$$

and also 
(2)

$$
\left\|\sum_{j} f_{j}\right\|_{\alpha} \leqslant K p
$$

whenever $f_{j} \in \Re$ is $B_{j}$-admissible, $j=1,2, \ldots$.

Proof. Fix $\varepsilon>0$, and choose a covering $\left\{D_{n}\right\}$ of $D \backslash X$ by discs with radii $\left\{r_{n}\right\}$ such that each $r_{n}$ is no greater than $s$, and

$$
\sum_{n} r_{n}^{1+\alpha}<M^{1+\alpha}(D \backslash X)+\varepsilon
$$

Then the $D_{n}$ cover each $B_{j} \backslash X$, and no $D_{n}$ meets more than $K_{1} p$ of the $B_{j}$. Thus

$$
\sum_{j} M^{1+\alpha}\left(B_{j} \backslash X\right) \leqslant K_{1} p \sum r_{n}^{1+\alpha} \leqslant K_{1} p\left\{M^{1+\alpha}(D \backslash X)+\varepsilon\right\} .
$$

This proves (1).

Now let $f_{j} \in \Re$ be $B_{j}$-admissible, $j=1,2, \ldots$ Fix $x, y \in \mathbf{C}$ and consider

$$
\left|f_{j}(x)-f_{j}(y)\right| /|x-y|^{\alpha} \text {. }
$$

We divide the integers $j$ into classes $F_{m}$, corresponding to $m=0,1,2,3, \ldots$, as follows. We say $j \in F_{m}$ if $m$ is the greatest integer not exceeding

$$
s^{-1} \min \left\{\operatorname{dist}\left(x, B_{j}\right), \operatorname{dist}\left(y, B_{j}\right)\right\} \text {. }
$$

Observe that the number of elements in $F_{m}$ does not exceed $K_{2} p m$.

For $m=0$ or 1 and $j \in F_{m}$ we use the crude estimate

$$
\left|f_{j}(x)-f_{j}(y)\right| /|x-y|^{\alpha} \leqslant\left\|f_{j}\right\|_{\alpha} \leqslant 1 .
$$

For $m>1, j \in F_{m}$ we consider two cases.

Case 1. $|x-y|>s$. Then

$$
\begin{aligned}
\frac{\left|f_{j}(x)-f_{j}(y)\right|}{|x-y|^{\alpha}} & \leqslant \frac{\left|f_{j}(x)\right|+\left|f_{j}(y)\right|}{s^{\alpha}} \\
& \leqslant \frac{K_{3} \gamma\left(B_{j}\right)\left\|f_{j}\right\|_{\alpha}}{(m s) s^{\alpha}} \text { by } \S 12 \\
& \leqslant \frac{K_{3} \gamma\left(B_{j}\right)}{m s^{1+\alpha}} .
\end{aligned}
$$

Case 2. $|x-y| \leqslant s$. Since $j \in F_{m}$ there is an arc $\Gamma$ joining $x$ to $y$ such that the length of $\Gamma$ does not exceed $6|x-y|$, and $\operatorname{dist}\left(\Gamma, B_{j}\right) \geqslant m s$. Thus

$$
\begin{aligned}
\frac{\left|f_{j}(x)-f_{j}(y)\right|}{|x-y|^{\alpha}} & =\frac{\left|\int_{\Gamma} f^{\prime}(z) d z\right|}{|x-y|^{\alpha}} \\
& \leqslant \frac{K_{4}|x-y|^{1-\alpha} \gamma\left(B_{j}\right)\left\|f_{j}\right\|_{\alpha}}{(m s)^{2}}<\frac{K_{4} \gamma\left(B_{j}\right)}{m^{2} s^{1+\alpha}} .
\end{aligned}
$$


Thus in either case

$$
\frac{\left|f_{j}(x)-f_{j}(y)\right|}{|x-y|^{\alpha}} \leq \frac{K_{5} M^{1+\alpha}\left(B_{j} \backslash X\right)}{s^{1+\alpha}} .
$$

Let $f=\Sigma_{j} f_{j}$. Then, abbreviating $M^{1+\alpha}\left(B_{j} \backslash X\right)=M_{j}$, we have

$$
\begin{aligned}
\frac{|f(x)-f(y)|}{|x-y|^{\alpha}} & \leqslant \sum_{j} \frac{\left|f_{j}(x)-f_{j}(y)\right|}{|x-y|^{\alpha}} \\
& \leqslant K_{6} p+\sum_{j} \frac{K_{5} M_{j}}{s^{1+\alpha}} \\
& \leqslant K_{6} p+K_{5} K_{1} p \text { by }(1) \\
& =K_{7} p .
\end{aligned}
$$

14. This lemma allows us to improve the estimate for $\|f\|_{u}$ of $\S 10$.

Corollary. Let $D$ be an open disc and let $f \in R(X)$ be $D$-admissible. Then

$$
\|f\|_{u} \leqslant K \gamma(D)^{\alpha /(1+\alpha)}
$$

Proof. In proving this we may assume that $X$ contains a neighbourhood of $3 D \backslash D$, and we do.

Cover the set of singularities of $f$ by discs $\frac{1}{2} B_{j} \subset D$ of side

$$
s=M^{1+\alpha}(D \backslash X)^{1 /(1+\alpha)}
$$

in such a way that no point belongs to more than 100 of the $B_{j}$. Choose functions $\varphi_{j} \in \mathscr{D}$ such that $0<\varphi_{j} \leqslant 1$, spt $\varphi_{j} \subset B_{j},\left\|\nabla \varphi_{j}\right\|_{u}<4 / s$, and $\Sigma \varphi_{j} \equiv 1$ on $\cup \frac{1}{2} B_{j}$, which is a neighbourhood of the set of singularities of $f$ (cf. [3]). Let $f_{j}=T_{\varphi_{j}} f$. Then $f=\sum f_{j}, f_{j} \in \tilde{\mathcal{R}}, f_{j}$ is analytic off $B_{j}$, and $f_{j}(\infty)=0$. Also $\left\|f_{j}\right\|_{\alpha} \leqslant K_{1}$ by the $T_{\varphi}$ estimate, so that $K_{1}^{-1} f_{j}$ is $B_{j}$-admissible.

Fix $z \in \mathbf{C}$, and divide the indices $j$ up into classes again: say $j \in G_{m}$ if $m$ is the greatest integer not exceeding $s^{-1} \operatorname{dist}\left(z, B_{j}\right)$. For $m>1$ and $j \in G_{m}$ we have

$$
\left|f_{j}(z)\right| \leqslant K_{2} \gamma\left(B_{j}\right) / m s
$$

by the Decay Lemma, $\$ 12$. Thus 


$$
\begin{aligned}
|f(z)| & \leqslant \sum_{j}\left|f_{j}(z)\right| \leqslant K_{3}\|f\|_{u}+\sum_{m=2}^{\infty} \sum_{j \in G_{m}}\left|f_{j}(z)\right| \\
& \leqslant K_{4}\left\{s^{\alpha}+\sum_{m=2}^{\infty} \sum_{j \in G_{m}} \frac{\gamma\left(B_{j}\right)}{m s}\right\}=K_{4} s^{\alpha}\left\{1+\sum_{m=2}^{\infty} \sum_{j \in G_{m}} \frac{\gamma\left(B_{j}\right)}{m s^{1+\alpha}}\right\} \\
& \leqslant K_{5} s^{\alpha}\left\{1+\left[\sum_{j} \frac{\gamma\left(B_{j}\right)}{s^{1+\alpha}}\right]^{1 / 2}\right\} \quad \text { (cf. }[6, \text { p. 201, 2.6]) } \\
& \leqslant K_{6} s^{\alpha}, \text { by } \S 13 \text { and } \S 15 .
\end{aligned}
$$

Thus $\|f\|_{u} \leqslant K_{6} s^{\alpha} \leqslant K_{7} \gamma(D)^{\alpha /(1+\alpha)}$.

15. We are now in a position to prove the sufficiency of the condition for approximation. In fact, we will prove a slightly stronger statement.

Suppose there exist constants $\mu>0, \tau>1$ such that for each point $x \in$ bdy $X$ and each disc $D$ centered at $X$,

$$
M^{1+\alpha}(\tau D \backslash X) \geqslant \mu M_{*}^{1+\alpha}(D \backslash \text { int } X) .
$$

Then $[\Re]_{\alpha}=A_{\alpha}(X)$.

Throughout the proof $K_{1}, K_{2}, K_{3}, \ldots$ stand for constants which may depend on $\alpha, \mu, \tau$ and $\|f\|_{\alpha}$, but not on any other variables.

Suppose $\mu$ and $\tau$ exist as in the statement. Then for each open disc $D$ of radius $r$ centered at a point of $\mathbf{C} \backslash$ int $X$ we have

$$
M^{1+\alpha}(\tau D \backslash X) \geqslant 4^{-1} \mu M_{*}^{1+\alpha}(D \backslash \operatorname{int} X),
$$

hence $\gamma(\tau D) \geqslant K_{1} \gamma_{A}(D)$ for each such disc $D$.

Fix $f \in A$. We shall prove that $f$ may be approximated in $\operatorname{Lip}(\alpha, X)$ norm by elements of $\tilde{R}$. First, we extend $f$ to $C$ so that the extension (also denoted by $f)$ lies in $\operatorname{lip}(\alpha, \mathrm{C})$ and is analytic off some disc. Fix $\delta>0$. Let $\left\{D_{n}\right\}_{1}^{\infty}$ be a covering of $\mathbf{C} \backslash$ int $X$ by open discs of radius $\delta$ centered at points of $\mathbf{C} \backslash$ int $X$ and such that no disc $D_{n}$ meets more than 100 others. Let $\left\{\varphi_{n}\right\}_{1}^{\infty} \subset \mathcal{D}$ be a sequence of functions such that $0 \leqslant \varphi_{n} \leqslant 1$, spt $\varphi_{n} \subset 2 D_{n},\left\|\nabla \varphi_{n}\right\|_{u} \leqslant 4 \delta^{-1}$, and $\Sigma_{1}^{\infty} \varphi_{n} \equiv 1$ on $\cup_{1}^{\infty} D_{n}$. Let $f_{n}=T_{\varphi_{n}} f$. Then $f_{n} \in A, f_{n} \equiv 0$ except for a finite number of indices $n$, and $f=\sum_{1}^{\infty} f_{n}$. Let $\eta(r)=r^{-\alpha} \omega_{f}(r)$, so that $\eta(r) \rightarrow 0$ as $r \downarrow 0$. For each $n, f_{n}$ is holomorphic off $2 D_{n}, f_{n}(\infty)=0$, and $\left\|f_{n}\right\|_{\alpha}<K_{2} \eta(\delta)$.

Now fix $n$ and, following Davie [3], let

$$
r=\frac{1}{2 \tau} \cdot \min \left\{\delta, M^{1+\alpha}(3 D \backslash X)^{1 /(1+\alpha)}\right\} .
$$

Cover the (closed) set of singularities of $f_{n}$ (a subset of $2 D_{n} \backslash$ int $X$ ) by centered discs $B_{j} \subset 2 D_{n}$ of radius $r$, in such a way that no point belongs to 
more than 25 of the $B_{j}$. Select a collection $\left\{\psi_{j}\right\} \subset \mathscr{D}$ of functions such that $0 \leqslant \psi_{j} \leqslant 1$, spt $\psi_{j} \subset 2 B_{j},\left\|\nabla \psi_{j}\right\|_{u} \leqslant 4 / r$, and $\sum \psi_{j} \equiv 1$ on $\cup B_{j}$, which is a neighbourhood of the set of singularities of $f_{n}$. Let $f_{j}^{*}=T_{\psi} f_{n}$. Then $f_{j}^{*} \in A, f_{j}^{*}$ is analytic off $2 B_{j}, f_{j}^{*}(\infty)=0,\left\|f_{j}^{*}\right\|_{\alpha} \leqslant K_{4} \eta(\delta)$, and $f_{n}=\Sigma_{j} f_{j}^{*}$. From the definition of $\gamma_{A}$ we deduce that

$$
\left|f_{j}^{* \prime}(\infty)\right| \leqslant K_{4} \eta(\delta) \gamma_{A}\left(2 B_{j}\right) \leqslant K_{5} \eta(\delta) \gamma\left(2 \tau B_{j}\right), \text { by hypothesis. }
$$

Thus there exist functions $g_{j}^{*} \in \mathcal{R}$ such that $g_{j}^{*}$ is analytic off $2 \tau B_{j}, g_{j}^{*}(\infty)=$ $0,\left\|g_{j}^{*}\right\|_{\alpha} \leqslant K_{5} \eta(\delta)$, and $g_{j}^{* \prime}(\infty)=f_{j}^{* \prime}(\infty)$. Let $g_{n}=\sum g_{j}^{*}$. Then $g_{n} \in \Re, g_{n}$ is analytic off $3 D_{n}, g_{n}(\infty)=0$, and $g_{n}^{\prime}(\infty)=f_{n}^{\prime}(\infty)$. Also, by Lemma 13 (2), $\left\|g_{n}\right\|_{\alpha} \leqslant K_{6} \eta(\delta)$.

We have

$$
\beta\left(f_{n}-g_{n}, D_{n}\right)=\sum_{j} \beta\left(f_{j}^{*}-g_{j}^{*}, D_{n}\right)=\sum_{j} \beta\left(f_{j}^{*}-g_{j}^{*}, G_{j}\right),
$$

since $f_{j}^{*}-g_{j}^{*}$ vanishes to second order at $\infty$. Hence by Lemma 11 and Lemma $13(1)$,

$$
\left|\beta\left(f_{n}-g_{n}, D_{n}\right)\right| \leqslant \sum_{j} K_{7} r \gamma\left(B_{j}\right) \eta(\delta) \leqslant K_{8} \gamma\left(2 D_{n}\right)^{(2+\alpha) /(1+\alpha)} \eta(\delta) .
$$

We may choose a function $h_{n} \in \Re$, analytic off $2 D_{n}$ and vanishing at $\infty$, with $\left\|h_{n}\right\|_{\alpha} \leqslant 2$ and $h_{n}^{\prime}(\infty)=\gamma\left(2 D_{n}\right)$. Forming

$$
k_{n}=g_{n}+\beta\left(f_{n}-g_{n}, D_{n}\right)\left(h_{n} / \gamma\right)^{2} \in \Re
$$

(where we have abbreviated $\gamma=\gamma\left(2 D_{n}\right)$ ), we deduce that

$$
\begin{aligned}
\left\|k_{n}\right\|_{\alpha} & \leqslant\left\|g_{n}\right\|_{\alpha}+\left|\beta\left(f_{n}-g_{n}, D_{n}\right)\right| \gamma^{-2}\left\|h_{n}^{2}\right\|_{\alpha} \\
& \leqslant K_{6} \eta(\delta)+K_{8} \gamma^{-\alpha /(1+\alpha)} \eta(\delta)\left\|h_{n}\right\|_{u} \leqslant K_{9} \eta(\delta)
\end{aligned}
$$

by Corollary 14. Also $k_{n}$ is analytic off $2 D_{n}, k_{n}(\infty)=0, k_{n}^{\prime}(\infty)=g_{n}^{\prime}(\infty)=$ $f_{n}^{\prime}(\infty)$, and $\beta\left(k_{n}, D_{n}\right)=\beta\left(g_{n}, D_{n}\right)+\beta\left(f_{n}-g_{n}, D_{n}\right)=\beta\left(f_{n}, D_{n}\right)$.

Let $q_{n}=f_{n}-k_{n}$. Then $f=\sum k_{n}+\sum q_{n}$. The first sum belongs to $\tilde{R}$. We will show that the second sum tends to zero in $\operatorname{Lip}(\alpha, C)$ norm as $\delta \downarrow 0$, so that $f \in[\tilde{\Re}]_{\alpha, c^{*}}$

Clearly $\left\|q_{n}\right\|_{\alpha} \leqslant K_{10} \eta(\delta)$, so that by Lemma $10,\left\|q_{n}\right\|_{u} \leqslant K_{11} \delta^{\alpha} \eta(\delta)$. Fix two distinct points $x, y \in \mathbf{C}$. In order to estimate

$$
|x-y|^{-\alpha}\left\{\sum q_{n}(x)-\sum q_{n}(y)\right\}
$$

we divide the indices $n$ into classes $F_{m}$, in the same way as in the proof of Lemma 13, with $s=2 \delta$. Thus $n \in F_{m}$ if $n s$ is the greatest integral multiple of $s$ not exceeding

$$
\min \left\{\operatorname{dist}\left(x, 2 D_{n}\right), \operatorname{dist}\left(y, 2 D_{n}\right)\right\}
$$

The number of indices in $F_{m}$ does not exceed $K_{11}(m+1)$. 
The function $q_{n}$ has a triple zero at $\infty$, so that $\delta^{-3}\left(z-c_{n}\right)^{3} q_{n}(z)$, the function, is analytic on $\Sigma \backslash 2 D_{n}$ (here $\left.c_{n}=c\left(D_{n}\right)\right)$. For $z \in \operatorname{bdy}\left(2 D_{n}\right)$,

$$
\left|\delta^{-3}\left(z-c_{n}\right)^{3} q_{n}(z)\right| \leqslant 8\left\|q_{n}\right\|_{u} \leqslant K_{12} \delta^{\alpha} \eta(\delta),
$$

hence by the maximum principle,

$$
\left|q_{n}(z)\right| \leqslant K_{13} \delta^{3+\alpha} \eta(\delta) d^{-3}
$$

whenever $d=\operatorname{dist}\left(z, 2 D_{n}\right)>s$.

If $k(z)$ is a bounded function, is analytic off a disc $D$ of radius $r$, and vanishes at $\infty$, and $0<R=\operatorname{dist}(z, D)$, then the uniform norm derivative decay estimate $[12$, p. 201$]$ states that

$$
\left|k^{\prime}(z)\right| \leqslant 4 r\|k\|_{u, \text { bdy } D} / R^{2} \text {. }
$$

If $d=\operatorname{dist}\left(z, D_{n}\right)>4 s$, take $D=\frac{1}{2} d D_{n}$, so that $\left\|q_{n}\right\|_{u, \text { bdy } D}<$ $K_{14} \delta^{3+\alpha} \eta(\delta) d^{-3}$ by (*), and conclude that

$$
\left|q_{n}^{\prime}(z)\right| \leqslant K_{15} \delta^{3+\alpha_{\eta}} \eta(\delta) d^{-4} .
$$

If $n$ belongs to one of the first six $F_{m}$ we use the crude estimate

$$
\left|q_{n}(x)-q_{n}(y)\right| /|x-y|^{\alpha} \leqslant\left\|q_{n}\right\|_{\alpha} \leqslant K_{6} \eta(\delta) .
$$

If $6 \leqslant m \in \mathbf{Z}$ and $n \in F_{m}$, we consider two cases.

Case $1 .|x-y| \leqslant s$. We have

$$
m s \leqslant \min \left\{\operatorname{dist}\left(x, 2 D_{n}\right), \operatorname{dist}\left(y, 2 D_{n}\right)\right\},
$$

so there is a curve $\Gamma$ joining $x$ to $y$, the length of which does not exceed $\pi|x-y|$, with the property that $\operatorname{dist}\left(\Gamma, 2 D_{n}\right)>m s$. Thus by $(* *)$,

$$
\begin{aligned}
\frac{\left|q_{n}(x)-q_{n}(y)\right|}{|x-y|^{\alpha}} & =\frac{1}{|x-y|^{\alpha}}\left|\int_{\Gamma} h_{n}^{\prime}(z) d z\right| \\
<\pi K_{15}|x-y|^{1-\alpha} s^{3+\alpha} \eta(\delta)(m s)^{-4} & <K_{16} \eta(\delta) m^{-4}
\end{aligned}
$$

Case 2. $|x-y|>s$. Then by (*),

$$
\begin{aligned}
\frac{\left|q_{n}(x)-q_{n}(y)\right|}{|x-y|^{\alpha}} & <\frac{\left|q_{n}(x)\right|+\left|q_{n}(y)\right|}{s^{\alpha}} \\
& <2 K_{13} s^{3+\alpha} \eta(\delta)(m s)^{-3}=K_{17} \eta(\delta) m^{-3} .
\end{aligned}
$$

Thus in either case 


$$
\begin{aligned}
& \frac{\left|\sum_{n} q_{n}(x)-\sum q_{n}(y)\right|}{|x-y|^{\alpha}} \leqslant \sum_{n} \frac{\left|q_{n}(x)-q_{n}(y)\right|}{|x-y|^{\alpha}} \\
& \quad \leqslant \sum_{m=0}^{5} \sum_{n \in F_{m}} K_{6} \eta(\delta)+\sum_{m=6}^{\infty} \sum_{n \in F_{m}} K_{18} \eta(\delta) m^{-3} \\
& \leqslant\left\{\sum_{m=0}^{5} K_{6} K_{11}(m+1)+\sum_{m=6}^{+\infty} K_{18} K_{11}(m+1) m^{-3}\right\} \eta(\delta) \\
& =K_{19} \eta(\delta) .
\end{aligned}
$$

Since $\eta(\delta) \rightarrow 0$ as $\delta \downarrow 0$, this proves that $\left\|\Sigma q_{n}\right\|_{\alpha} \rightarrow 0$ as $\delta \downarrow 0$, so we are done.

16. As a special case we obtain a characterisation of those compact sets $X$ on which all $f \in \operatorname{lip}(\alpha, X)$ may be approximated in $\operatorname{Lip}(\alpha, X)$ norm by rational functions.

COROLlaRY. A necessary and sufficient condition that

$$
[\Re]_{\alpha}=\operatorname{lip}(\alpha, X)
$$

is that there exist $\mu>0$ such that $M^{1+\alpha}(D \backslash X) \geqslant \mu r^{1+\alpha}$ for every open disc $D$ of radius $r(0<\alpha<1)$.

This follows from our theorem because $M_{*}^{1+\alpha}(D)=(2 r)^{1+\alpha}$.

17. Corollary. If $X$ has zero area and $0<\alpha<1$, then

$$
[\Re]_{\alpha}=\operatorname{lip}(\alpha, X) \text {. }
$$

Proof. Let $D$ be any disc of radius $r$. Then, denoting Lebesgue measure on the plane by $m$, we have $m(D \backslash X)=m(D)=\pi r^{2}$. Let $\left\{B_{j}\right\}$ be a covering of $D \backslash X$ by discs with radii $\left\{r_{j}\right\}, r_{j} \leqslant r$. Then

$$
\sum r_{j}^{1+\alpha}>\frac{\sum r_{j}^{2}}{r^{1-\alpha}} \geqslant \frac{m(D \backslash X)}{\pi r^{1-\alpha}}=r^{1+\alpha},
$$

hence $M^{1+\alpha}(D \backslash X)>r^{1+\alpha}$. Thus the condition of Corollary 16 is satisfied, with $\mu=1$.

J. Garnett has shown the author how to give a direct constructive proof of this fact. There is also an entirely different proof, based on duality.

18. Corollary. If $0<\alpha<1$ and $M_{*}^{1+\alpha}($ bdy $X)=0$, then

$$
[\Re]_{\alpha}=\operatorname{lip}(\alpha, X) \cap A(X) \text {. }
$$

Proof. If $E_{1}$ and $E_{2}$ are two subsets of $\mathbf{C}$, then

$$
M_{*}^{1+\alpha}\left(E_{1} \cup E_{2}\right)<M_{*}^{1+\alpha}\left(E_{1}\right)+M_{*}^{1+\alpha}\left(E_{2}\right) \text {. }
$$

This is an immediate consequence of the definition of $M_{*}^{\beta}$ and the subadditivity of $M_{h}$. It follows that 


$$
\begin{aligned}
M_{*}^{1+\alpha}(D \backslash \text { int } X) & \leqslant M_{*}^{1+\alpha}(\text { bdy } X)+M_{*}^{1+\alpha}(D \backslash X) \\
& \leqslant M_{*}^{1+\alpha}(\text { bdy } X)+M^{1+\alpha}(D \backslash X),
\end{aligned}
$$

hence if $M_{*}^{1+\alpha}($ bdy $X)=0$, then the condition of our theorem is satisfied, with $\mu=1$.

The condition $M_{*}^{1+\alpha}(E)=0$ is equivalent to $\oiint^{1+\alpha}(E)<\infty$, where $\oiint^{1+\alpha}$ is $(1+\alpha)$-dimensional Hausdorff measure [5, (2.10)].

19. Before giving some examples, we need a definition. Let $B(x, r)$ denote the disc $\{z \in \mathbf{C}:|z-x| \leqslant r\}$. If $E \subset \mathbf{C}$ and $\beta>0$, then the $\beta$-dimensional upper density of $E$ at the point $x \in \mathbf{C}$ is defined as

$$
\lim \sup \frac{M^{\beta}(E \cap B(x, r))}{r^{\beta}} ;
$$

the lower density is the corresponding lim inf, and in case these two coincide, we refer to the density.

20. Example. We construct a compact set $X \subset \mathbf{C}$ such that $X$ is the closure of its interior, and $[\Re]_{u}=A(X)$, but $[\Re]_{\alpha} \neq A_{\alpha}(X)$.

Fix $\beta, \alpha<\beta<1$. We begin with a closed square $P$, and inside $P$ an arc $\Gamma$ having positive $(1+\beta)$-dimensional lower density at each of its points [7]. We then remove from $P$ a sequence of thin wavy open strips $S_{1}, S_{2}$, $S_{3}, \ldots$, so that the $S_{j}$ "accumulate" only on $\Gamma$ and accumulate at every point of $\Gamma$, and so that $\cup_{j} S_{j}$ has zero $(1+\alpha)$-dimensional density at each point of $\Gamma$. Then we set $X=P \backslash\left(\cup_{j} S_{j}\right)$. For any small disc $D$ of radius $r$ about any point of $\Gamma, M_{*}^{1+\alpha}(D \backslash$ int $X)$ will be bounded below by some constant times $r^{1+\alpha}$, whereas $M^{1+\alpha}(D \backslash X)$ will be $o\left(r^{1+\alpha}\right)$. So the condition of the theorem cannot hold for any $\mu>0$. Thus $[\Re]_{\alpha} \neq A_{\alpha}(X)$. Since the diameters of the components of $\mathbf{C} \backslash X$ are bounded away from zero, it follows that $[\Re]_{u}=$ $A(X)$ (cf. [6, p. $219(8.3)])$.

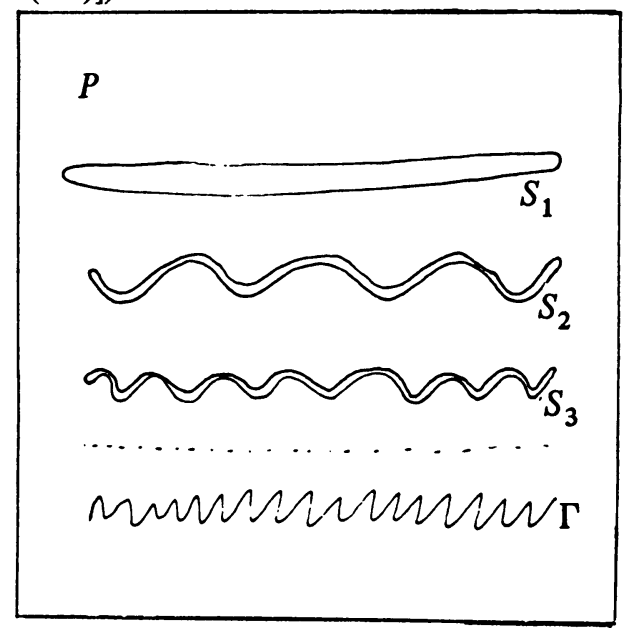

FIGURE 1 
21. ExAmple. We construct a set $X$ with empty interior such that the analytic polynomials $\mathcal{P}$ are uniformly dense in $C(X)$, but $[\Re]_{\alpha} \neq \operatorname{lip}(\alpha, X)$.

Choose a sequence of positive numbers $l_{n}$ such that $\Sigma_{1}^{\infty} l_{n}^{\alpha}<1$. Then $\Sigma_{1}^{\infty} l_{n}<1$ and we may form a Cantor set $C$ of positive length on $[0,1]$ by deleting successively (open) intervals of length $l_{n}$. Let $\lambda$ denote Lebesgue measure on the line.

LEMMA. $[0,1] \backslash C$ has zero $\alpha$-dimensional density at $\lambda$ almost all points of $C$.

Proof. Let $\left(a_{n}, b_{n}\right)$ be the interval of length $l_{n}$ in $[0,1] \backslash C$. Then by Fubini's Theorem,

$$
\begin{aligned}
\int_{0}^{1} \sum_{n=1}^{\infty} \frac{l_{n}^{\alpha}}{\left|z-a_{n}\right|^{\alpha}} d \lambda(z) & =\sum_{n=1}^{\infty} l_{n}^{\alpha} \int_{0}^{1} \frac{d \lambda(z)}{\left|z-a_{n}\right|^{\alpha}} \\
& \leqslant 2^{\alpha}(1-\alpha)^{-1} \sum_{n=1}^{\infty} l_{n}^{\alpha}<\infty,
\end{aligned}
$$

so that

$$
\sum_{n=1}^{\infty} \frac{l_{n}^{\alpha}}{\left|z-a_{n}\right|^{\alpha}}<\infty
$$

for $\lambda$ almost all $z \in[0,1]$. Similarly,

$$
\sum_{1}^{\infty} \frac{l_{n}^{\alpha}}{\left|z-b_{n}\right|^{\alpha}}<\infty
$$

for $\lambda$ almost all $z \in[0,1]$. For $z \in C$ the upper $\alpha$ density of $[0,1] \backslash C$ at $z$ is

$$
\lim \sup \frac{M^{\alpha}([z-r, z+r] \backslash C)}{r^{\alpha}} \leqslant \limsup \frac{\Sigma^{\prime} l_{n}^{\alpha}}{r^{\alpha}}
$$

(where the sum is taken over those $n$ for which $\left[a_{n}, b_{n}\right]$ meets $[z-r, z+r]$ ).

$$
\begin{aligned}
& \underset{r \downarrow 0}{\lim \sup } \Sigma^{\prime}\left\{\frac{l_{n}^{\alpha}}{\left|z-a_{n}\right|^{\alpha}}+\frac{l_{n}^{\alpha}}{\left|z-b_{n}\right|^{\alpha}}\right\} \\
& \leqslant \underset{r \downarrow 0}{\lim \sup } \sum_{N_{r}}^{\infty}\left\{\frac{l_{n}^{\alpha}}{\left|z-a_{n}\right|^{\alpha}}+\frac{l_{n}^{\alpha}}{\left|z-b_{n}\right|^{\alpha}}\right\} \\
& \text { (where } N_{r} \text { is the first index in } \Sigma^{\prime} \text { ) } \\
& =0
\end{aligned}
$$

for $\lambda$ almost all $z \in C$. This proves the lemma.

Now set $X=C \times[0,1]$. Then $[\mathcal{P}]_{u}=C(X)$ by Mergelyan's Theorem [6], since $X$ does not separate the plane. But clearly $\mathbf{C} \backslash X$ has zero $(1+\alpha)$ density at $\mathfrak{L}^{2}$ almost all points of $X$, so $[\Re]_{\alpha} \neq \operatorname{lip}(\alpha, X)$ by Corollary 16 . 
22. Example. The term Swiss Cheese is traditionally applied to any compact set $X$ obtained by removing from the closed unit disc an infinite sequence $\left\{D_{n}\right\}$ of disjoint open discs, with radii $\left\{r_{n}\right\}$ and centres $\left\{a_{n}\right\}$, such that $\Sigma r_{n}<1$ and $\cup_{n} D_{n}$ is dense in the unit disc. For any such $X,[\Re]_{u} \neq$ $C(X)[1],[6]$, and hence a fortiori $[\Re]_{\alpha} \neq \operatorname{lip}(\alpha, X)$, for $0<\alpha<1$.

Fix $0<\alpha<1$. A larger class of cheeses is obtained by relaxing the condition on the radii of the excised discs to $\sum r_{n}^{1+\alpha}<\infty$. We call such a cheese an " $\alpha$-cheese". If $X$ is an $\alpha$-cheese, then $[\Re]_{\alpha} \neq \operatorname{lip}(\alpha, X)$. To see this, note that by Fubini's Theorem,

$$
\begin{aligned}
\int_{X} \sum_{1}^{\infty} \frac{r_{n}^{1+\alpha}}{\left|z-a_{n}\right|^{1+\alpha}} \operatorname{am}(z) & =\sum_{1}^{\infty} r_{n}^{1+\alpha} \int \frac{d m(z)}{\left|z-a_{n}\right|^{1+\alpha}} \\
& \leqslant \sum_{1}^{\infty} r_{n}^{1+\alpha} 2 \pi(1-\alpha)^{-1}<\infty .
\end{aligned}
$$

Hence

$$
\sum_{1}^{\infty} \frac{r_{n}^{1+\alpha}}{\left|z-a_{n}\right|^{1+\alpha}}<\infty \quad \text { a.e. } d m .
$$

For $m$ almost all such $z$, it follows that

$$
M^{1+\alpha}(B(z, r) \backslash X) / r^{1+\alpha} \rightarrow 0
$$

as $r \downarrow 0$. Precisely speaking, the limit is zero for any $z$ for which the series converges, unless $z$ happens to belong to bdy $D_{n}$ for some $n$. This is seen by essentially the same argument as that of the last section.

Thus the necessary condition for rational approximation is violated, and so $[\Re]_{\alpha} \neq \operatorname{lip}(\alpha, X)$.

23. We close with some remarks about polynomial approximation. Let $\mathscr{P}$ denote the space of analytic polynomials. It is not hard to see that $[R]_{\alpha, X}=$ $[\mathscr{P}]_{\alpha, X}$ if and only if $\mathbf{C} \backslash X$ is connected. Thus $[\mathscr{P}]_{\alpha, X}=A_{\alpha}(X)$ if and only if C $\backslash X$ is connected and there exists a constant $\mu>0$ such that

$$
M^{1+\alpha}(D \backslash X) \geqslant \mu M_{*}^{1+\alpha}(D \backslash \text { int } X)
$$

whenever $D$ is an open disc. Also $[\mathscr{P}]_{\alpha, X}=\operatorname{lip}(\alpha, X)$ if and only if $\mathbf{C} \backslash X$ is connected and there exists a constant $\mu>0$ such that

$$
M^{1+\alpha}(D \backslash X) \geqslant \mu r^{1+\alpha}
$$

whenever $D$ is an open disc and the radius of $D$ is $r$.

\section{REFERENCES}

1. Andrew Browder, Introduction to function algebras, Benjamin, New York, 1969. MR 39 \#7431. 
2. Lennart Carleson, Selected problems on exceptional sets, Van Nostrand Math. Studies, no. 13, Van Nostrand, Princeton, N.J., 1967. MR 37 \#1576.

3. A. M. Davie, Analytic capacity and approximation problems, Trans. Amer. Math. Soc. 171 (1972), 409-444. MR 50 \#2502.

4. E. P. Dolženko, On removal of singularities of analytic functions, Uspehi Mat. Nauk 18 (1963), no. 4(112), 135-142; English transl., Amer. Math. Soc. Transl. (2) 97 (1971), 33-41. MR 27 \# 5898; 42 \#5740.

5. Herbert Federer, Geometric measure theory, Springer-Verlag, New York, 1969. MR 41 \#1976.

6. T. Gamelin, Uniform algebras, Prentice-Hall, Englewood Cliffs, N.J., 1969.

7. T. W. Gamelin and John Garnett, Pointwise bounded approximation and Dirichlet algebras, J. Functional Analysis 8 (1971), 360-404. MR 45 \#4135.

8. J. Garnett, Analytic capacity and measure, Lecture Notes in Math., vol. 297, SpringerVerlag, Berlin and New York, 1972.

9. L. I. Hedberg, The Stone-Weierstrass theorem in Lipschitz algebras, Ark. Mat. 8 (1969), 63-72. MR 41 \#5973.

10. M. S. Mel'nikov, Metric properties of analytic $\alpha$-capacity and approximation of analytic functions with Hölder condition by rational functions, Mat. Sb. (N.S.) 79 (121) (1969), 118-127 = Math. USSR Sbornik 8 (1969), 115-124. MR 42 \#3287.

11. E. M. Stein, Singular integrals and differentiability properties of functions, Princeton Univ. Press, Princeton, N.J., 1970. MR 44 \# 7280.

12. A. G. Vituškin, The analytic capacity of sets in problems of approximation theory, Uspehi Mat. Nauk 22 (1967), no. 6(138), 141-199 = Russian Math. Surveys 22 (1967), no. 6, 139-200. MR 37 \#5404.

Department of Mathematics, University of California, Los Angeles, California 90024

Current address: Department of Mathematics, Maynooth College, County Kildare, Ireland 